\title{
High Speed Optimum WSN Routing using Modified Distance Measure based Clustering: HSO-SAA
}

\author{
Priyanka Chourey \\ Bansal Institute of Research \& Technology \\ Bhopal, India
}

\author{
Imran Ali Khan \\ Bansal Institute of Research \& Technology \\ Bhopal, India
}

\begin{abstract}
In the recent times wireless sensors network (WSN) are opted for acquiring the sensor data to improve the performance. Sleep and awake based routing is efficient way of increasing the life of networks which is restricted by power availability of sensors. But conventional sleep aware and awake based methods are relatively computationally slow. Therefore, this paper proposes a modified absolute distance measure for clustering based routing. It is proposed to enhance the execution speed and energy efficiency of the conventional protocol. Therefore, this paper proposes to design an efficient routing algorithm HSO-SAA for the WSN networks via examination and testing of numerous state of art routing protocols related to WSN. The optimum parametric selection approach is used for increasing energy efficiency of routing protocol. Various experimentations had been performed in order to evaluate the performance of proposed optimum design along with existing clustering based protocols. Performance is compared based on the network life time, alive nodes, latency as FND, and the number of packets transmitted. Overall proposed energy model is good enough to improve the energy uses of WSN.
\end{abstract}

\section{General Terms}

Wireless Sensor Network

\section{Keywords}

Energy Efficiency,, Wireless Sensor network, Clustering, Routing Protocols, Heterogeneity. Network Lifetime

\section{INTRODUCTION}

Recently lot of researchers are enthusiastically trying the build and deploy WSN networks for data acquisition. As sensor node acts as a switch in the network thus, assurance of efficient routing becomes the essential part to prolonging the utilization of energy. A WSN consists of the assortment of huge number of tiny hubs (nodes), which are also working as switches in the network. These nodes are having limited energy sources as non chargeable and non-replaceable in real time. it is mandatory to enhance the energy efficiency of these WSN systems. The sleep aware and awake method is widely used for saving the energy of the WSN networks. This forces energy utilization as a massive research field in WSN. Energy protection may directly impact to improve the lifetime of the networks. This paper addresses the prime issues of lifetime improvement for WSN routing design. Basic concept adopted in paper is to improve the energy model of the network nodes.

The major contributions of the paper are to enhance the network life and throughput and data uses by porting the optimum design parameters associated to improvement in network energy. It is proposed to modify the distance measure between nodes and cluster heads $(\mathrm{CH})$

\subsection{Role of WSN}

WSN is a network of tiny, independent hardware units called the sensors, which are deployed to monitor the physical or ecological conditions used for monitoring the temperature, vibration, acoustic, environment humidity, current, weight, movements, health, and corrosion [1]. These sensors nodes are deployed to gather the real time data from the unrealistic earth environments and transmit it to centralised base station (BS). This BS has the capability to analyze the physical existence of place where the accumulated data belongs to based on the supportive WSN applications [2] [3]. WSN contains massive sensor points for sending the information between sensors to sensor (S2S) or to the central BSs. Various WSN applications are described in next section for understanding the requirement of work. Paper first validates the existing EESAA protocol and basic energy adaptive LEACH protocols evaluated for comparing the performance. Then final phase designs the optimum parametric selection based O-EESAA protocol. Performance is measured in terms of latency defined as first node died count, network lifetime and the counts of the cluster heads $(\mathrm{CHs})$ to study the routing protocols.

\subsection{Structure of WSN}

The basic structure of the WSN networks along with broad application areas look like as given in Figure 1. There is a huge area or field covered by distributed sensor nodes. The nodes are classified as centralized BS and the normal sensor nodes.

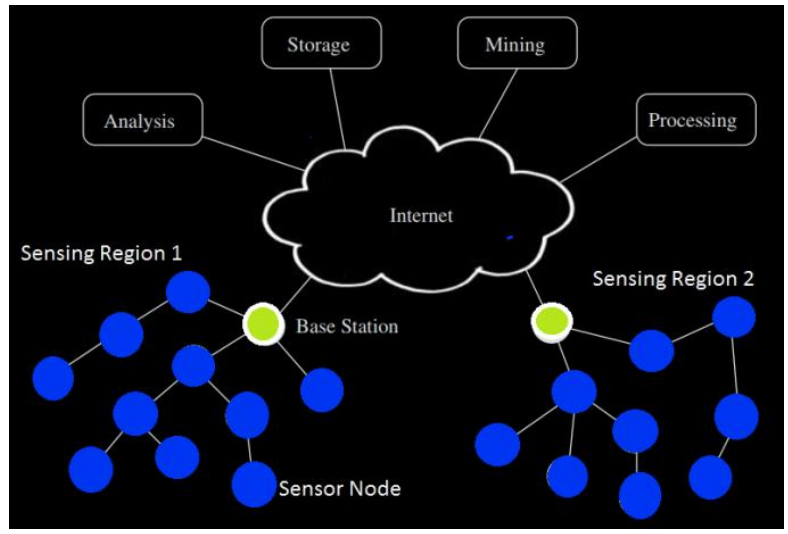

Figure 1 Structure of WSN and applications

The internet is used as the network for the connectivity of all the nodes with the high end application servers. The application filed is broadly being classified as data Analysis, Memory storage, data mining application, and the data processing for classification tasks. Routing is a method of sending the data through optimum path to application server. Wireless sensor networks (WSNs) are represented as a special form of Adhoc networks kind. WSN represent an electro- 
computing technology having an exponential success in the logistical, military, and scientific areas. It not only realizes benefits for the customer in a sophisticated way, but in addition provides this with high flexibility.

\section{LITTERATURE REVIWE}

There has lot of work done for increasing energy efficiency of the heterogeneous networks. Usually this paper focused on the optimizing the design parameter based on investigation of the clustering based protocols using Sleep Aware and Awake approach. The primarily paper reviewed the work done for WSN protocol designing of energy efficient clustering based protocols. The basic classification of the various clustering based WSN routing protocol methodologies are given in the Figure 2. .

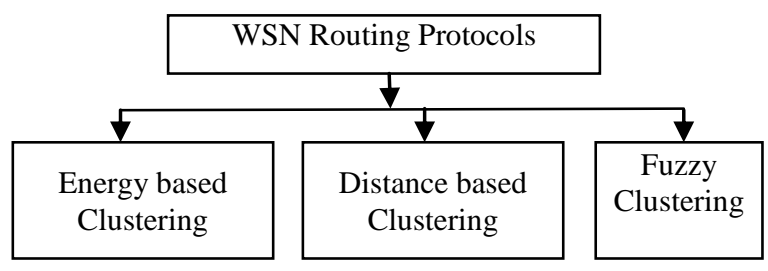

Figure 2 Classification of Routing Protocols

Ansam Ennaciri et al [1] have designed the efficient energy balancing based method using the sleep aware and awake (EESAA) methods. T. Shah et al [2] have first time proposed an new energy efficient routing protocol approach using sleep awake and aware basis for improving the energy performance. The protocol takes advantage of remaining energy and the distance measure of the nodes for clustering and $\mathrm{CH}$ selection. Priya et al [3] have designed the enhanced DEEC protocol using the clustering and distributed energy network. Initially they have used two tire normal and advance nodes for energy distribution therefore improves the performance in terms of lifetime. Akshay Verma et al [4] proposed the fuzzy logic based routing method using effective clustering called as (FLEC) for homogeneous wireless sensor networks has been introduced for Mobile Sink. A fuzzy based protocol is used to extend the network life time. Khurana et al [5] have proposed new cluster head selection based LEACH-MAC algorithm designed for the WSN.

Komalpreet Kaur et al [6] have designed the enhanced protocol performance of the distributed energy efficient based routing using clustering (EDEEC). They have introduced additional supper node structure for improving the protocol lifetime and energy performance. R. Saxena et al [7] hate presented the good performance evaluation of three different routing protocols and has proposed the modified version of the basic SEP called TSEP performs better then EESAA and LEACH.

A. Kaur et al [12] have proposed a new optimized protocol using the geographical adoptive fidelity concept based on sleep/awake based energy efficient routing protocol. They proposed that this concept with GAF significantly improve the performance and parametric optimization can improve the performance better. The summary of literature review is given in the Table 1. The current paper is focused to improve energy and lifetime performance of the existing EESAA protocol
Table 1 Summary of Review of Routing protocols

\begin{tabular}{|c|c|c|}
\hline Authors & Methodology & Approach \\
\hline $\begin{array}{l}\text { Ansam } \\
\text { Ennaciri } \\
{[1]}\end{array}$ & $\begin{array}{l}\text { Designed an energy } \\
\text { balancing approach for } \\
\text { EESAA protocol }\end{array}$ & $\begin{array}{l}\text { Used multi layer } \\
\text { balancing method for } \\
\text { performance } \\
\text { improvement in } \\
\text { energy of EESAA }\end{array}$ \\
\hline $\begin{array}{l}\text { Priya } \\
\text { et al [3] }\end{array}$ & $\begin{array}{l}\text { The improved the } \\
\text { energy performance of } \\
\text { the DEEC }\end{array}$ & $\begin{array}{l}\text { They have used an } \\
\text { extra supper nodes as } \\
\text { the third node tire }\end{array}$ \\
\hline $\begin{array}{l}\text { R. Saxena } \\
\text { et al [7] }\end{array}$ & $\begin{array}{l}\text { TSEP protocol for } \\
\text { perform better over } \\
\text { other protocols based } \\
\text { on clustering }\end{array}$ & $\begin{array}{l}\text { Stable election based } \\
\text { protocol for the } \\
\text { performance } \\
\text { evaluation. }\end{array}$ \\
\hline \begin{tabular}{l|} 
Sercan \\
Vançin et al \\
{$[12]$}
\end{tabular} & $\begin{array}{l}\text { Design a three tire an } \\
\text { energy based efficient } \\
\text { routing }\end{array}$ & $\begin{array}{l}\text { balanced method of } \\
\text { threshold sampling }\end{array}$ \\
\hline Proposed & $\begin{array}{lr}\text { Optimum network } \\
\text { parameters design for } \\
\text { extended lifetime of } \\
\text { EESAA }\end{array}$ & $\begin{array}{l}\text { Modified distance for } \\
\text { node clustering for } \\
\text { high speed protocol } \\
\text { design }\end{array}$ \\
\hline
\end{tabular}

\section{THEORY OF EESAA PROTOCOL AND VALIDATION}

The size of the sensors is an important limitation, mainly in terms of energy autonomy and lifetime because the battery must be very small. For this reason, many studies are currently focusing on managing the energy consumed by the sensors in the network. Keeping this in mind Energy Efficient Sleep Awake Aware (EESAA) protocol [5] had proposed an algorithm to improve the quality of service (QoS) based on an efficient clustering approach. Authors have implemented the simulation in MATLAB for proving the efficiency of their EESAA algorithm in which the performance of our algorithm is evaluated and compared with available clustering protocols. The EESAA is a load balancing protocol improves the $\mathrm{CHs}$ selection technique, by choosing $\mathrm{CHs}$ according to the remaining energy of the nodes. This paper is expected to improve the speed of the existing SAA protocols

\section{PROPOSED MODIFIED ABSOLUTE DISTANCE}

Paper proposes a sleep awake based cluster head $(\mathrm{CH})$ selection method using the nodes energy constipation and there distance from base station (BS). The distance measure plays the significant role in the WSN designing. Existing routing protocols tried to reduce energy consumption based on sleep-awake for the nodes away from the BS. In conventional LEACH protocols [5] the lifetime of network is limited since nodes far away from the BS consume more energy. The major contributions of the paper are to enhance the network life and intermission of data. It is possible by opting the optimum network design parameters. In order to achieve balanced energy consumption, distance of a node to its base station should also be taken into consideration.

In the basic EESAA [2] protocol concept of node pairing is introduced. In conventional sensor nodes in the application having the minimum distance between them are proposed to form the pair of node for data communication.

The nodes are supposed to switch Sleep and Awake during the communication intervals. A node in a pair switches to awake mode if their distance from BS is less than its pair node. An awake node gathers data from all surroundings and transmits it to $\mathrm{CHs}$. Initially all nodes are assigned initial energy $\mathrm{E}_{0}$. The initial $\mathrm{CH}$ election threshold $\mathrm{Th}$ is defined as; 
$\mathrm{Th}=$

$\left\{\begin{array}{cc}\frac{P_{d}}{1-P_{d^{*}}\left(\left(\text { Rou }_{1}\right) \bmod \frac{1}{P_{d}}\right.} & \text { if } n \in A \\ 0 & \text { else }\end{array}\right.$

The total energy $\mathrm{E}_{\mathrm{T}}$ dissipates by $\mathrm{CH}$ during transmitting the data to the respective BS is: calculated as

$$
E_{T}=E_{T X} \times L_{A} \times E_{a m p} \times L_{A} \mathrm{x}
$$

$D_{B S}^{2}$

Where $\mathrm{L}_{\mathrm{A}}$ is the aggregate data and the $D_{B S}$ is the distance between the $\mathrm{CH}$ and BS. Therefore, it is observed that the overall energy dissipation depends directly on the distance measure.

Square Euclidean distance measure defined between points, $\mathrm{a}$ and $\mathrm{b}$, having the $\mathrm{k}$ dimensions is mathematically defined as;

$\sum_{j=1}^{k}\left(a_{j}-b_{j}\right)^{2}$

In the previous versions of the protocols [1, and 2], the distance between nodes are calculated by Euclidean distance defined as;

$$
D_{(i, j)}=\operatorname{sqrt}\left(\left(S(i)_{\cdot x d}-\left(S(j) \cdot_{x d}\right)\right)^{2}+(S(i) \cdot y d-(S(j) \cdot y d))^{2}\right)
$$

In this paper for improving the execution time per rounds a modified absolute distance measure is proposed for calculating the distance between nodes. The modified distance measures the

$\operatorname{MAD}_{(\mathrm{i}, \mathrm{j})}=\operatorname{abs}\left(\left(\left(\mathrm{S}(\mathrm{i})_{\cdot \mathrm{xd}}-\left(\mathrm{S}(\mathrm{j})_{\mathrm{xd}}\right)\right)+\left(\mathrm{S}(\mathrm{i})_{\mathrm{yd}}-\left(\mathrm{S}(\mathrm{j})_{\mathrm{yd}}\right)\right)\right)\right)$

The rest of protocol process is similar to the [Ref. 2]. The modified distance measure MAD as defined is expected to improve the execution speed. But it is approximate distance therefore, it may compromise the slight life time and therefore parametric optimization is essentially required to improve the performance. Thus in this paper optimum experimentation parameter are selected as presented in the next section.

\section{OPTIMUM HUGH SPEED PROTOCOL (HSO-SAA)}

Therefore in order to increase the network lifetime in this paper an extended protocol designed is called as HSO-SSA. Where, SAA represents the Sleep Aware and Awake approach. The network simulation parameters are optimally selected to enhance the energy utilization of the network. The parameter for the optimum node pairing and un-pairing is set to $\mathrm{x}=\mathrm{y}=3$. Where the $\mathrm{x}$ is the pairing parameter and $\mathrm{y}$ is the un-pairing parameter. Calculated for the heterogeneous energy as in equation 6 defined as

$$
\mathrm{S}(\mathrm{i}) . \mathrm{E}=\mathrm{Eo}
$$

The distance measure between the nodes and $\mathrm{CH}$ are calculated using the modified absolute distance measure. It is observed that proposed modifications made significant improvement in performance of protocol. Values of optimum initial transmitted and received energy and probability of elections of node and node distribution coefficients for the OEESAA protocol is given in the Table 2 below.

Table 2 node distribution and design parameters for HSO-

\begin{tabular}{|c|c|c|}
\hline Eo & Initial energy in $\mathrm{J}$ & $0.8 \mathrm{~J}$ \\
\hline$E_{Y x}=E_{E x}$ & $\begin{array}{l}\text { Transmitted and received } \\
\text { energy }\end{array}$ & $50 \mu \mathrm{J}$ \\
\hline$E_{f s}$ & Free space energy & $10 \mathrm{pJ}$ \\
\hline$E_{D A}$ & Data aggregation energy & $5 \mu \mathrm{J}$ \\
\hline A & $\begin{array}{l}\text { Energy enhancement } \\
\text { friction }\end{array}$ & 2.5 \\
\hline$r_{\max }$ & $\begin{array}{lll}\begin{array}{l}\text { Maximum number of } \\
\text { rounds }\end{array} & \\
\end{array}$ & 9000 \\
\hline $\mathrm{X}$ & nodes pairing friction & 3 \\
\hline $\mathrm{Y}$ & Nodes un-pairing friction & 3 \\
\hline
\end{tabular}
SAA protocol

\begin{tabular}{|l|l|l|}
\hline Variable & Notation & Value \\
\hline $\mathrm{N}$ & Number of the nodes & 100 \\
\hline$P$ & $\begin{array}{l}\text { Initial probability of } \\
\text { cluster heads }\end{array}$ & 0.2 \\
\hline
\end{tabular}

Validation of protocols

In the Figure 3 the validation of protocols for the live nodes performance of EESAA protocols are presented. It can be seen that EESAA works better but life is short.

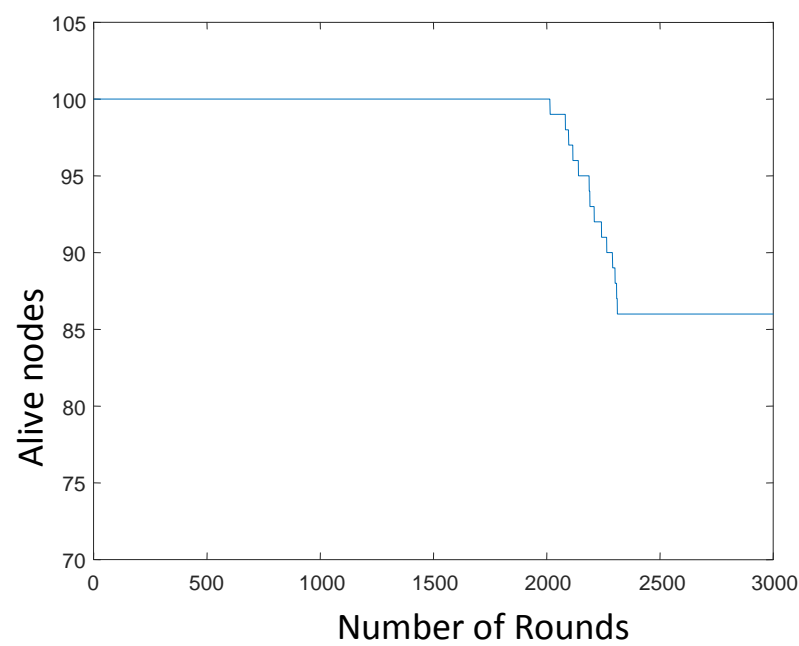

Figure 3 Validation of EESAA protocol alive nodes

\section{Results and Discussion}

This paper proposed the modified absolute distance measure based an optimum routing protocol as HSO-SAA and compares the performance of the proposed energy efficient protocols using the optimum parametric design. Results are presented for the comparative simulations with similar clustering based SEP and EESAA protocols. Different experiments have been performed on core 2 Duo $2.4 \mathrm{GHz}$ processor in MATLAB software. As the distance is approximate measure therefore, it gives the advantage of modelling process for a real system.

Initially the parametric optimization for enhance performance of the existing routing protocol is required to evaluate. To analyze and compare the performance of the existing protocol and proposed parametric optimization the live node performance is compared in the Figure 4. It can be observed that the optimum O-EESAA performs better than the existing EESAA protocol in tires of live nodes and throughput. 


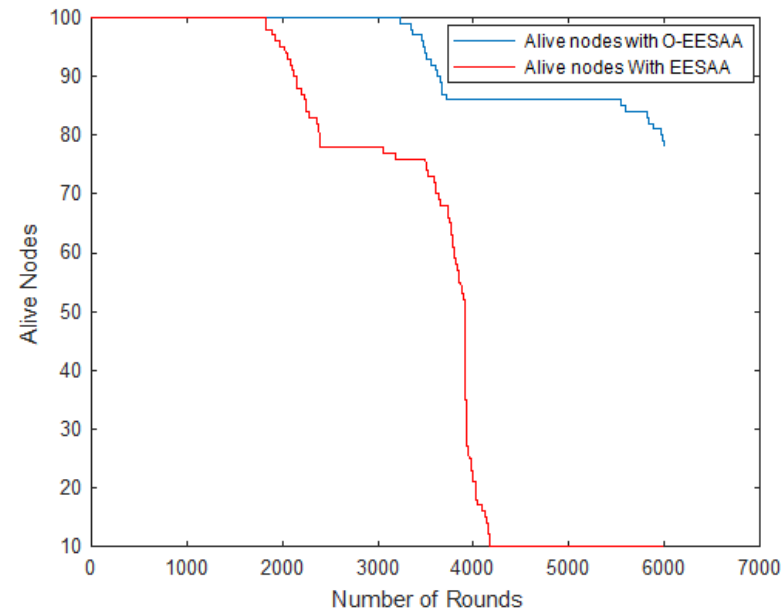

Figure 4 Comparison of the alive nodes for EESAA and $\mathrm{O}$-EESAA Protocol for WSN

\section{Experiment 1: Comparison of HSO-SAA vs. EESAA}

The proposed modified distance based high speed routing protocol HSO-SAA is compared with the existing EESAA and SEP protocol. The performance is evaluated for number of live nodes in Figure 5. For this experiment $\mathrm{x}$ and $\mathrm{y}$ are scaled by 3 and energy enhancement factor $A=2.5$. The initial energy is set to $0.8 \mathrm{~J}$. It can be observed that the HSO-SAA protocol performs better than the existing EESAA protocol.

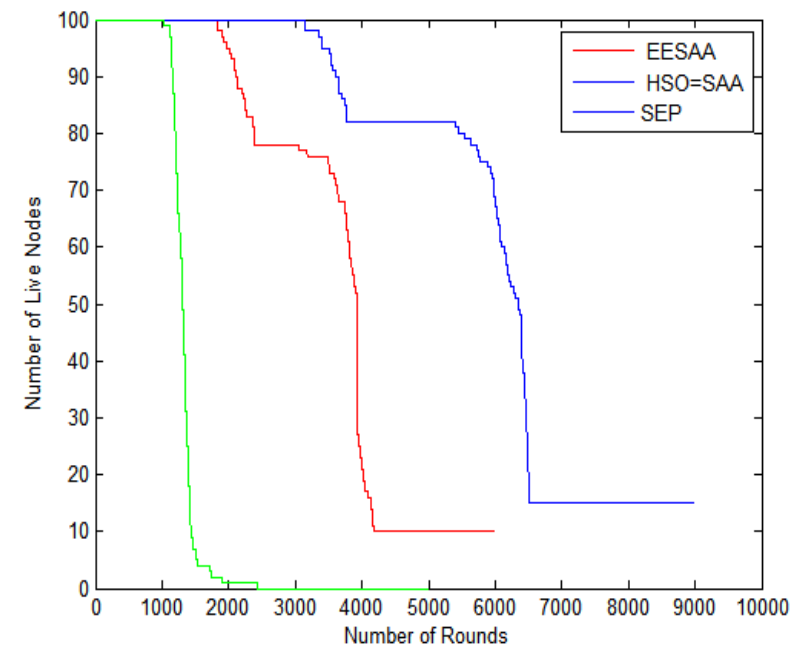

Figure 5 Comparison of the live nodes for proposed HSO-SAA protocol for WSN

It can be observed that the lifetime of the network is enhanced by around $40 \%$ compared to existing protocol. And the complete network is died at around $30 \$$ more rounds comparatively using HSOSAA.

\section{Experiment 2. No. of CH Counts for proposed protocol}

In this section the number of $\mathrm{CH}$ counts fir the existing and the proposed routing protocols are compared. The performance comparison is shown in the Figure 6. The proposed routing protocol with high speed optimum parameters routing enhances the length of the $\mathrm{CH}$ counts and distribute over the long time to enhance the energy efficiency.

The number of packets transmitted to BS and to the $\mathrm{CH}$ for the existing and the proposed routing protocols are compared respectively in the Figure 7 and the Figure 8 . It is observed that the overall transmission efficiency become almost doubled with proposed routing protocol

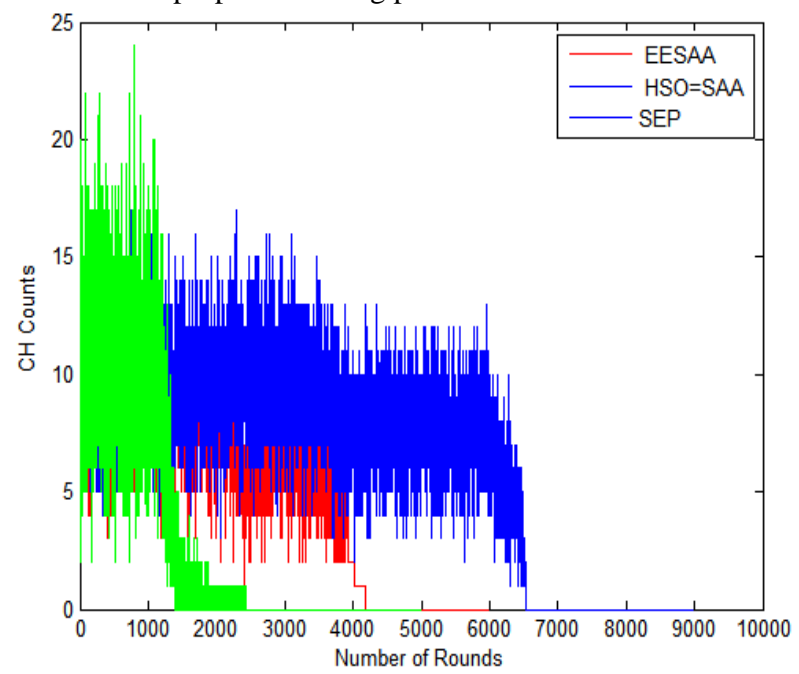

Figure 6 Comparison of live nodes for SEP, EESAA and proposed

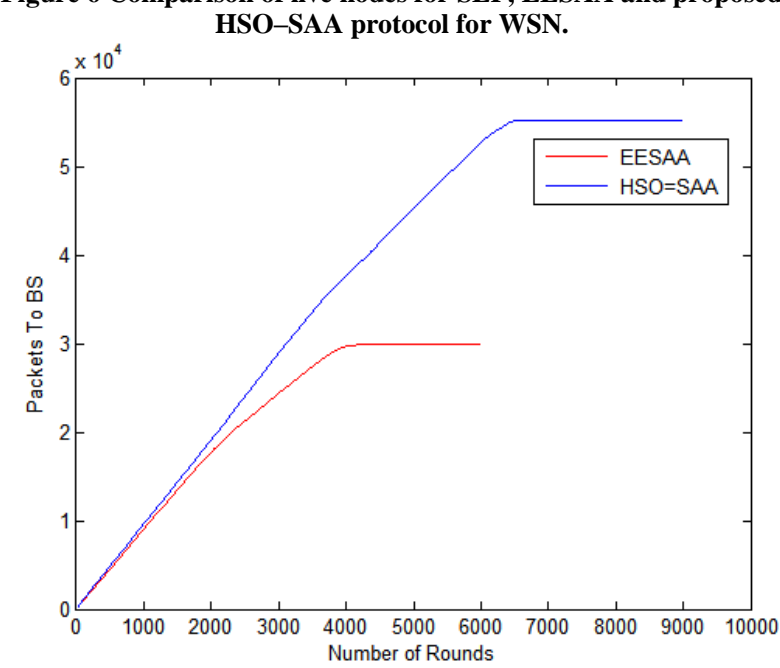

Figure 7 Number of packets transmitted to BS

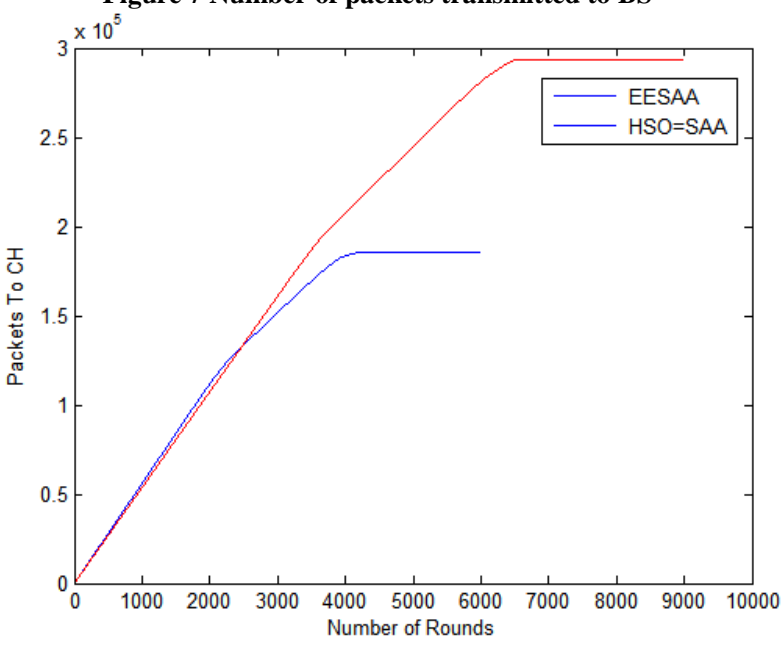

Figure 8 Number of packets transmitted to $\mathrm{CH}$

HSO-SAA protocol

It can be observed that propped protocol increases the execution speed significantly. The time is expected to vary for each execution as the networks are randomly defined. Therefore the high speed performance of HSO-SAA is satisfied. This is because of modified distance measures. 


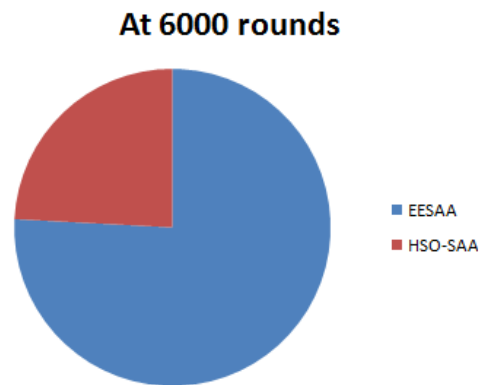

Figure 9 Comparison of the elapsed time for proposed

\section{CONCLUSION AND FUTURE SCOPE}

The WSN is essential for the area of IOT and requirement of energy efficient routing is always a challenging task. Paper proposed to design the improved performance of the EESAA protocol. The goal was to design the energy efficient routing protocol and performance comparison with the state of art SEP, and EESAA protocols. The proposed HSO-SAA protocol transmits 400 more packets to BS compared to EESAA protocol. Similarly the lifetime in terms of the FND is improved by around $41 \%$ by using the HSO-SAA protocol compared to EESAA. And the speed is also improved significantly due to modified proposed distance measure. This emphasize that proposed protocol is more energy efficient compared to all other protocols mentioned here. Over all paper contributes significant improvement in energy performance and lifetime of the WSN.

\section{ACKNOWLEDGMENT}

Authors acknowledge to all who have supported the research to make a success on their individual capacity. All the referred authors are also acknowledged by authors.

\section{REFERENCES}

[1] Ansam Ennaciri, Mohammed Erritali, Jamaa Bengourram "Load Balancing Protocol (EESAA) to improve Quality of Service in Wireless sensor network", Elsevier International Workshop on Web Search and Data Mining (WSDM) April 29 - May 2, 2019

[2] T. Shah, N. Javaid and T. N. Qureshi, "Energy Efficient Sleep Awake Aware (EESAA) intelligent Sensor Network routing protocol," 2012 15th International Multitopic Conference (INMIC), Islamabad, pp. 317322,2012

[3] Priya EDEEC-Enhanced Distributed Energy Efficient Clustering Protocol for Heterogeneous Wireless Sensor Network (WSN) Scheme for WSN, IEEE Access 2018

[4] Akshay Verma , Sunil Kumar, Prateek Raj Gautam , Tarique Rashid, and Arvind Kumar, Fuzzy Logic Based Effective Clustering of Homogeneous Wireless Sensor Networks for Mobile Sink", IEEE Sensors Journal, Vol. 20, No. 10, May 15, 2020

[5] Khurana, B., P., \& Kant, K., "LEACH-MAC: A newcluster head selection algorithm for wireless sensor networks", in Journal of Wireless Networks, 22, 49-60

[6] Komalpreet Kaur, Er. Shivani Sharma." Enhanced Distributed Energy Efficient Clustering Protocol", International Conference on Computer Communication and Informatics (ICCCI -2020), Jan. Coimbatore, INDIA $22-24,2020$

[7] R. Saxena, V. Rishiwal and O. Singh, "Performance
Evaluation of Routing Protocols in Wireless Sensor Networks," 2018 3rd International Conference On Internet of Things: Smart Innovation and Usages (IoTSIU), Bhimtal, pp. 1-6, 2018

[8] Kassim MR, M.; Harun, A.N. Applications of WSN in agricultural environment monitoring systems. In Proceedings of International Conference on Information and Communication Technology Convergence (ICTC), Jeju Korea, 19-21 October 2016

[9] Georgios Smaragdakis Ibrahim Matta Azer Bestavros, "SEP: A Stable Election Protocol for clustered heterogeneous wireless sensor networks", Second International Workshop on Sensor and Actor Network Protocols and Applications (SANPA 2004).

[10] Dionisis Kandris, Christos Nakas, Dimitrios Vomvas and Grigorios Koulouras, "Applications of Wireless Sensor Networks: An Up-to-Date Survey", MDPI journal of applied system information, Vol. 3, Issue 14, 2020

[11] Amee Vishwakarma, Prof. Papiya Dutta, "A Review on Various Energy Efficient Clustering Protocols of WSN" in Journal of Emerging Technologies and Innovative Research (JETIR) Volume 4, Issue 082017

[12] A. Kaur and R. Gujral, "Optimized GAF protocol based sleep/awake protocol for WSN to improve Network Lifetime," 2016 3rd International Conference on Signal Processing and Integrated Networks (SPIN), Noida, pp. 411-416, 2016

[13] Zantalis, F.; Koulouras, G.; Karabetsos, S.; Kandris, D. A review of machine learning and IoT in smart transportation, Future Internet Vol. 11, Issue 94, 2019

[14] Ch. Usha Kumari and Tatiparti Padma, "Efficient data gathering algorithm in wireless sensor networks with optimal path mobile sink. Journal of Wireless Communications and Mobile Computing, 2015

[15] Sahraei, S. H., Kashani, M. M. R., Rezazadeh, J., \& Farah Bakhsh, R. (2018). Efficient job scheduling in cloud computing based on genetic algorithm. International Journal of Communication Networks and Distributed Systems, 22, 447-467

[16] Farhadian, F., Kashani, M. M. R., Rezazadeh, J., FarahBakhsh, R., \& Sandrasegaran, K. (2019). An efficient IoT cloud energy consumption based on genetic algorithm. Digital Communications and Networks

[17] Tyagi S, Kumar N. "A systematic review on clustering and routing techniques. Based upon LEACH protocol for wireless sensor networks", Journal of Network and Computer Applications, Elsevier, Vol. 36, issue 2, 2013,pp 623-645.

[18] Kumar, V., Jain, S. \& Tiwari, S. (2011). Energy efficient clustering algorithms in wireless sensor networks: A survey. IJCSI international Journal of Computer Science Issues, 8(5), 2

[19] Sharma, A. K., \& Kourtney, H., "Hybrid energy efficient distributed protocol for heterogeneous wireless sensor network". International Journal of Computer Applications, 4(6), 1-5. 2010.

\section{AUTHOR'S PROFILE}

Priyanka Chourey is an research scholor at the Bansal institute of research \& technology Bhopal. She has got the 
becholoers degree in computer science enginnering from sagar institute of research and technology bhopal in 2013. Now she is pursuing the Master of Technology form the Bansal Institute of Research \& Technology Bhopal.

Prof. Imran Ali Khanhe has received the Bachelor degree in
Computer Science engineerng. He has persuing Ph.D. degree from VIT Bhopal, India. He is currently working in Department of Computer science Engineering, at Bansal Institue of Research and Technoogy Bhopal. He has more then a decade of teaching experience and guided many M.Tech students. 